\title{
The Sandwich Supratrochlear Artery Perforator Flap with the Pericranium for Reconstruction of Full-Thickness Nasal Defects
}

\author{
TAREK SHOUKR, M.D.*; MOHAMED O. TOMOUM, M.D.** and MOHAMED SADAKA, M.D.* \\ The Departments of Plastic \& Reconstructive Surgery* and Otolaryngology/Head \& Neck Surgery**, Faculty of Medicine, \\ Tanta University
}

\begin{abstract}
Introduction: Repair of the nasal defects is one of the most challenging areas for the reconstructive surgeons due to the lack of uniform skin thickness, complex contours, and small close subunits. Our aim is to utilize the bipaddle forehead flap with a cartilage graft in between for simultaneous cutaneous and lining reconstruction of the hemi-nasal defect after excision of the basal cell carcinoma.
\end{abstract}

Patients and Methods: Our study included 25 patients with full thickness hemi-nasal defects after excision of basal cell carcinoma. We utilized the sandwich forehead flap which is bipaddle (cutaneous and periosteal layers) based on the deep and superficial perforator branches of the supratrochlear artery respectively, and cartilage graft in between.

Results: Age of the patients ranged from 40 to 65 years (mean age of 51 years). With a follow-up period ranged from 6 to 18 months (mean of 11 months), no flap necrosis nor cartilage exposure were observed. Twelve cases only came back for debulking.

Conclusion: The bipaddle forehead flap with intervening cartilage graft offers a good option for reconstruction of full thickness hemi-nasal defect with no extradonor site morbidity.

Key Words: Nose - Esthetics - Cutaneous neoplasm - Perforator flap - Forehead.

\section{INTRODUCTION}

In addition to its important functional role, the nose plays a significant esthetic unit of the face. Nasal defects could be acquired by trauma, inflammatory processes, and cutaneous neoplasms [1]. Repair of the nasal defects is one of the most challenging areas for the reconstructive surgeon due to the lack of uniform skin thickness, complex contours, and small close subunits [2].

Regardless of the etiology of the defect, the primary goals of the repair would be restoration of the nasal appearance and function. Full thickness nasal defects will require not only cutaneous and lining reconstruction of the defect, but also cartilage or bone grafts to provide nasal contour and support, and to prevents flap contraction and improves esthetic outcomes. Vascularized tissues are required on both surfaces of the graft material [3-8].

Large or complex defects will require a paramedian forehead flap for reconstruction along with cartilaginous and/or bony support, as well as a lining flap. These lining flaps could be harvested from vestibular mucosa [9]. However, the absence of these mucosal flaps creates a challenge to provide adequate vascularized lining and cover tissue simultaneously. Staged procedures, the use of a second local flap, or free tissue transfer to provide adequate lining have been described by previous studies [6-8]. The anterior-based pericranial flap $(\mathrm{PF})$ is widely used in anterior skull base reconstructive surgery with a high success rate [10,11]. The pericranial flap is supplied by the deep perforator branches of the supratrochlear artery [12]. In this study we describe a sandwich supratrochlear artery perforator flap (cutaneous and periosteal layers) with intervening cartilage to provide simultaneous cutaneous and lining reconstruction of the hemi-nasal defects at the time of initial repair after excision of basal cell carcinoma.

\section{PATIENTS AND METHODS}

Fig. (1 A-D):

This study was conducted between August 2016 to March 2019 on 25 cases with full thickness hemi-nasal defects affecting most of the esthetic nasal subunits and the nasal support after excision of basal cell carcinoma. A handheld doppler was used to identify the supratrochlear artery and its 
path was marked on the overlying skin. A standard paramedian forehead flap was designed along the course of the supratrochlear artery [13]. The distal end of the skin component of the flap remained within the hairless part of the forehead skin. The exact shape of the acquired defect was transferred to the distal end of the skin component of the flap using a template. After excision of the skin tumor and complete hemostasis, efforts were directed to reconstruction of the nose. An incision was made along the outline of the skin component of the flap and through the subcutaneous tissue layer. In the subgaleal plane, the skin adjacent and superior to the flap was undermined without incising the pericranium. Before elevation of the pericranium a pocket for the graft material between the skin flap and pericranium is created, this dissection between the skin and the pericranium was $2 \mathrm{~cm}$ above the orbital rim in the supratrochlear artery area to preserve the superficial branches of the skin component of the flap [12].

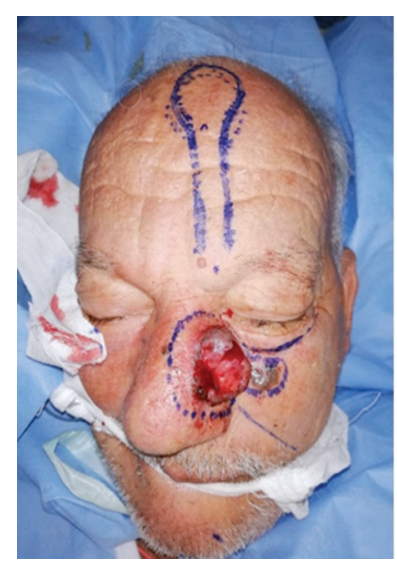

(A)

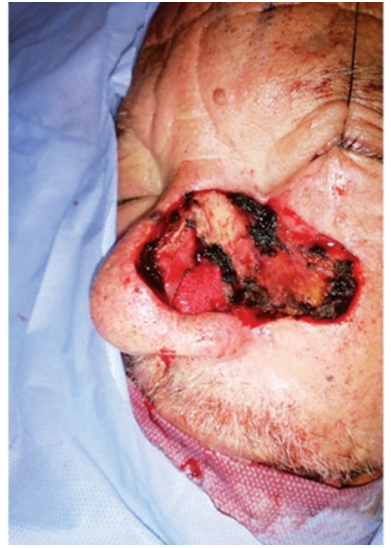

(B)

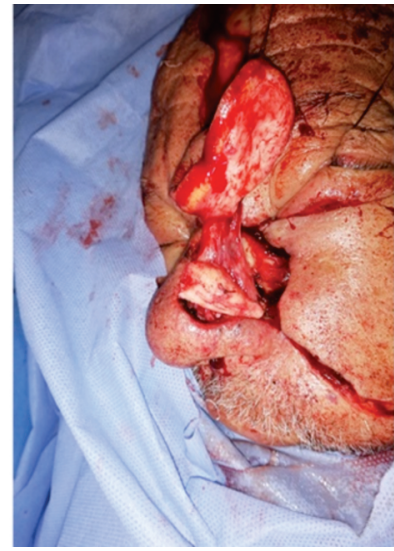

(C)

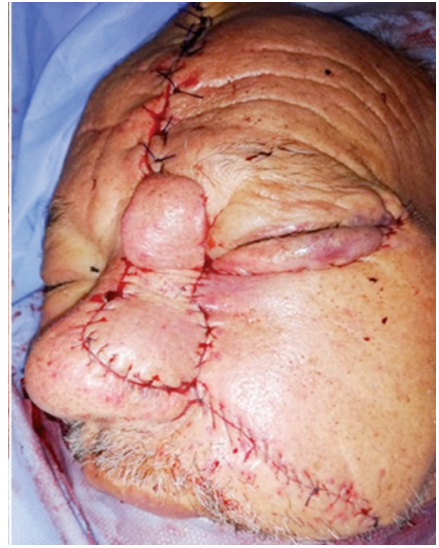

(D)

Fig. (1): "A-D": "A" Marking the flap, "B" Excision of the tumor, "C" The bilaminar flap (Skin and pericranium) with a cartilage graft in between, " $\mathrm{D}$ " Closure of both the repient and donor sites.

\section{RESULTS}

This study was conducted between August 2016 to March 2019 on 25 cases with basal cell carcinoma of the nose. The age ranged between 40 and 65 years (with a mean age of 51). Seventeen cases were males and 8 cases were females. The follow up period ranged from 6 to 18 months with a mean of 11 months. No recurrence observed during the follow-up period, no flap necrosis or cartilage exposure was observed in any case. Postoperative flap congestion was observed in 5 cases from which only one case required some sutures release while the others resolved spontaneously as demonstrated in Fig. (2).

Twelve cases only came back for debulking and revision. Routine nasal endoscopy was done for all patients after one month to document the degree of mucosalization, which was successful in all cases as shown in Fig. (3). 

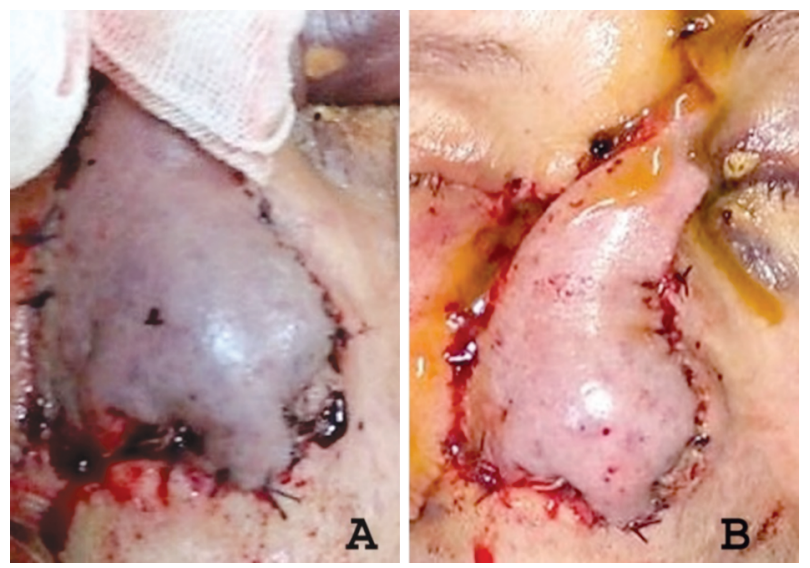

Fig. (2): Postoperative flap congestion that resolved spontaneously.

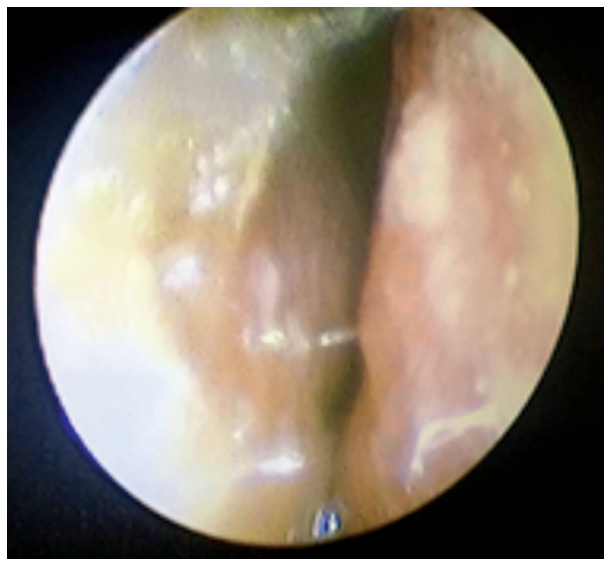

Fig. (3): Nasal endoscopy showing mucosalization of the pericranium.
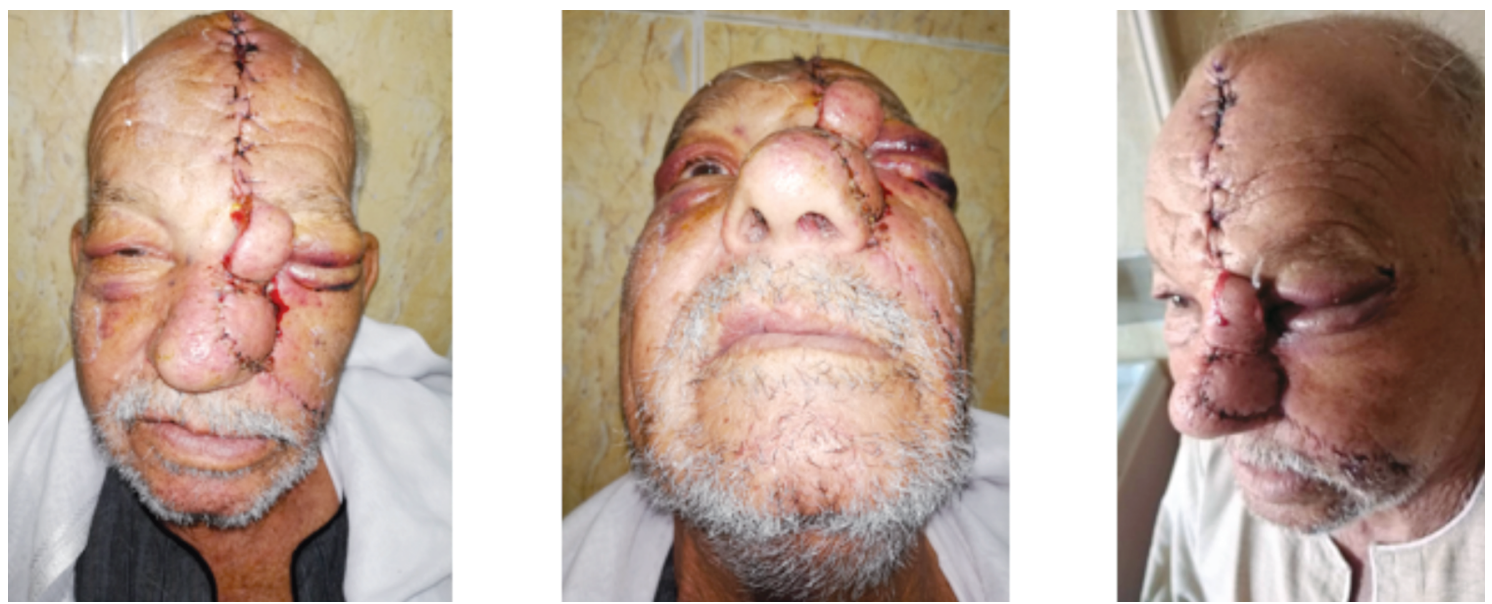

Fig. (4): Same patient two weeks postoperative.

\section{DISCUSSION}

The paramedian forehead flap dates to the Ayur Veda of Sushruta in $800 \mathrm{BC}$ India, who used it to reconstruct the external nose following punitive amputation $[4,15]$. Western acceptance of the technique was delayed in 1816, Carpue was the first one to perform the technique in the Western world by reporting 2 successful cases of pedicled forehead flap nasal reconstruction $[4,15]$. In 1800s, cicatricial contracture of unlined flaps and its effects on the external morphology and airway patency became in concern [4]. The era of folding the distal end of the flap in onto themselves to provide lining soon followed but with inferior esthetic results due to thickness of tissue, absence of alar margins sharpness, lack of nasal tip definition and inability to accurately place primary graft material. Menick reported in detail the relationship between nasal support and lining [6]. Skin grafting and vestibular/ septal mucosal flaps are simple and effective techniques for lining the forehead skin flap to recon- struct small full-thickness nasal defects providing that the septum is intact to maintain the nasal support. Large nasal defects with loss of the septum and destruction of the cartilage skeleton require a vascularized lining tissue to assure primary placement of supporting graft material and inhibiting tissue contracture.

Cheek or nasolabial turn-in flaps, free tissue transfer and pericranial flaps can provide these vascularized lining tissue. The drawacks of the turn-in flaps have been mentioned. The free tissue transfer flap significantly increases the difficulty, the time, and cost of the operative procedure. Free tissue transfer and turn-in flaps increase the number of donor sites and therefore patient morbidity, while pericranial flaps offer the advantage of being within the same donor site of the covering skin component [1].

Pericranial flaps have been widely used for the reconstruction of various facial defects. These flaps consist of the periosteum of the skull and an over- 
lying connective tissue with excellent vascularity and blood supply mainly from supratrochlear artery when based anteriorly $[\mathbf{1 2}, \mathbf{1 6}]$. Brackley and Jones [17] reported the use of the pericranial flap for replacement of the lining tissue for reconstruction of a full-thickness defect of the nasal dorsum using the skin and pericranium as separate flaps based on the same vessels allowing the primary placement of a cartilage graft to support the side wall of the nose, they also reported epithelialization of the lining flap during the follow-up period. In this study we report the use of the sandwich supratrochlear perforator flap for full-thickness nasal defects. The flap is bilaminar, providing a pericranial lining layer and skin covering layer based on the deep and superficial perforator branches of the supratrochlear artery respectively. This flap design has several advantages: Elevation of the forehead flap and pericranium as a single entity with creation of a pocket in between to fit the cartilage graft preserve the galeal perforators with flap vascularity, no need for additional incisions, and without increasing the size of the forehead defect the scalp can be undermined superiorly through the same donor site to harvest the pericranium. However, debulking may be needed to obtain better esthetic results. In this study we limited the defect to the heminasal size so we can only use unilateral flap to cover the defect to focus on assessment of the flap viability and possible donor site morbidity, but theoretically we can use bilateral flaps to cover total nasal defects with the expected donor site morbidity that should be entitled in a separate study to evaluate costs and benefits for the patient.

\section{Conclusion:}

Bipaddle forehead flap with a cartilage graft in between is a good option for the reconstruction of the full thickness nasal defect. The pericranial layer provides a well-vascularized lining tissue that will support primary placement of structural graft, without increasing the difficulty of the procedure or morbidity to the patient.

\section{Compliance with ethical standards:}

The authors declare that they have no conflicts of interest to disclose.

Approval was obtained from the Local Ethical Committee of Tanta University, Faculty of Medicine. Written informed consent in Arabic language was taken for every patient.

\section{REFERENCES}

1- Potter J.K., Ducic Y. and Ellis E.: Extended Bilaminar Forehead Flap with Cantilevered Bone Grafts for Reconstruction of Full-Thickness Nasal Defects. J. Oral Maxillofac. Surg., 63: 566-570, 2005.

2- Wolfswinkel E.M., Weathers W.M., Cheng D. and Thornton J.F.: Reconstruction of Small Soft Tissue Nasal Defects. Semin Plast. Surg., 27 (2): 110-116, 2013.

3- Menick F.J.: A 10-year experience in nasal reconstruction with the three-stage forehead flap. Plast. Reconstr. Surg., 109: 1856-61, 2002.

4- Menick F.J.: Esthetic refinements in use of the forehead for nasal reconstruction: The paramedian forehead flap. Clin. Plast. Surg., 17: 607-22, 1990.

5- Danahey D.G. and Hilger P.A.: Reconstruction of large nasal defects. Otolaryngol. Clin. North Am., 34: 695-711, 2001.

6- Menick F.J.: The use of skin grafts for nasal lining. Clin. Plast. Surg., 28: 311-21, 2001.

7- Rohrich R.J., Barton F.E. and Hollier L.: Nasal reconstruction, in Aston S.J. (ed): Plastic Surgery. Philadelphia, PA, Lippincott-Raven, pp 513-528, 1997.

8- Muzaffar A.R. and English J.M.: Nasal reconstruction. Selected readings. Plast. Surg., 9 (13): 1-25, 2009.

9- Austin G.K. and Shockley W.: Reconstruction of Nasal Defects: Contemporary Approaches. Curr. Opin. Otolaryngol. Head Neck Surg., 24 (5): 453-460, 2016.

10- Balagopal P.G., George N.A., Ajith R., et al.: Anterior skull base reconstruction after cranio-facial resections using galeal pericranial flap. Gulf J. Oncolog., 1: 84-88, 2014.

11- Paloma V., Samper A. and Cervera-Paz F.J.: Surgical technique for reconstruction of the nasal septum: The pericranial flap. Head Neck, 22: 90-94, 2000.

12- Sertel S. and Pasche P.: Pericranial Flap for Inner Lining in Nasal Reconstruction. Annals of Plastic Surgery, 77 (4): 425-432, 2016.

13- Mathes S.J. and Nahai F.: Median forehead flap, in Mathes S.J., Nahai F. (eds): Reconstructive Surgery: Principles, Anatomy, and Technique. New York, NY, Churchill Livingstone, pp 260-270, 1997.

14- Lee M., Callahan S. and Cochran S.: Auricular cartilage: Harvest technique and versatility in rhinoplasty. American Journal of Otolaryngology, 32 (6): 547-552, 2011.

15- Anita N.H. and Daver B.M.: Reconstructive surgery for nasal defects. Clin. Plast. Surg., 8: 535-63, 1981.

16- Argenta L.C., Friedman R.J., Dingman R.O., et al.: The versatility of pericranial flaps. Plast. Reconstr. Surg., 76: 695-702, 1985.

17- Brackley P.T. and Jones N.S.: The use of a periosteal/forehead flap with sandwiched conchal cartilage graft: A novel approach for nasal reconstruction in the absence of a nasal septum. Plast. Reconstr. Surg., 110: 831-5, 2002. 